\title{
Quantitation of IL-2Rp75 (CD122) expression on mononuclear cells in rheumatic disease
}

\author{
Paul Creamer, Nicolas D Hall
}

\begin{abstract}
Objective-To compare expression of the p75 chain of the interleukin-2 receptor (IL-2Rp75, CD122) on peripheral and synovial mononuclear cells in rheumatoid and non-rheumatoid inflammatory arthritis.

Methods-Peripheral blood (PBMC) and synovial (SFMC) mononuclear cells were isolated from subjects with rheumatoid arthritis $(n=16)$ and non-rheumatoid inflammatory arthritis $(n=12)$. PBMC were isolated from six healthy controls. Expression of CD122 was examined using indirect immunofluorescence and quantitative flow cytometry.

Results-There was no difference in IL-2Rp75 expression on PBMC from rheumatoid arthritis patients, nonrheumatoid arthritis patients, and controls. In subjects with rheumatoid arthritis there was no difference in IL-2Rp75 expression on PBMC and SFMC. However, in the non-rheumatoid arthritis group there was an increase in IL-2Rp75 expression on SFMC compared with PBMC $(P=0.0032)$. On SFMC there was a greater expression of IL-2Rp75 in non-rheumatoid arthritis than in rheumatoid arthritis $(P=0.0007)$. Expression was greater on CD8 positive cells and in subjects with shorter duration of disease. Conclusions-The p75 chain of the IL-2 receptor, an important $T$ cell activation antigen, is not upregulated in synovial fluid. This appears to be a disease specific defect and provides further support for the concept of "frustrated" or incomplete $T$ cell activation in this disease. Key terms: IL-2 receptor; rheumatoid arthritis; flow cytometry
\end{abstract}

(Ann Rheum Dis 1996;55:844-847)

Rheumatology Unit, Bristol Royal Infirmary, Bristol BS2 8HW, United Kingdom P Creamer

Bath Institute for Rheumatic Diseases and School of Pharmacy and Pharmacology, University of Bath Bath BA2 7AY, United Kingdom N D Hall

Correspondence to: Dr N D Hall.

Accepted for publication 10 July 1996

The role of $T$ cells in rheumatoid synovitis remains controversial. The most widely accepted model proposes presentation of an "arthritogenic peptide" in the context of certain HLA class II specificities, resulting in expansion of antigen specific $T$ cells, production of $T$ cell derived cytokines, and a chronic inflammatory destructive synovitis. This view is supported by a number of observations and has been reviewed elsewhere, ${ }^{1}$ though the degree to which it is responsible for established synovitis has been challenged. $^{2}$

The most potent stimulus to $T$ cell expansion is interleukin-2 (IL-2). The high affinity IL-2 receptor (IL-2R) is formed by a complex between at least three molecules including the p55 chain (CD25), the p75 chain (IL-2Rp75, CD122), and the recently identified third subunit, the $\gamma$ chain. Despite the presence of other activation markers, only a minority of synovial fluid mononuclear cells are positive for IL-2R, at least when measured using an antibody directed against the IL-2Rp55 chain. ${ }^{3}$ This, combined with findings of defective IL-2 secretion in rheumatoid arthritis and hyporesponsiveness of rheumatoid arthritis synovial fluid cells to mitogenic stimuli, ${ }^{4}$ has given rise to the concept of "frustrated activation" of synovial fluid T cells. ${ }^{3}$

The larger p75 subunit has not been studied in rheumatoid arthritis, though the fact that some cells do not express IL-2Rp55 yet are capable of responding to $\mathrm{IL}-2^{6}$ and that IL-2Rp75 contains the functional domain for signal transduction ${ }^{6}$ suggests that it may be of importance.

IL-2 is known to stimulate resting $\mathrm{T}$ cells to produce $\gamma$ interferon in the absence of antigen and without a proliferative response. ${ }^{7}$ This effect is almost certainly mediated through the p75 receptor. $T$ cells from rheumatoid arthritis patients fail to produce normal levels of $\gamma$ interferon in response to $\mathrm{IL}-2^{8}$ though they respond normally to allogeneic cells, suggesting that the responsiveness to IL-2 rather than the synthetic capacity for $\gamma$ interferon is reduced in rheumatoid arthritis. One explanation of this defect, which appears to be disease specific, would be downregulation of IL-2 receptors.

The aim of this study was to test the hypothesis that IL-2Rp75 is specifically downregulated in rheumatoid arthritis. This was done by quantitating expression of IL2Rp75 on peripheral mononuclear cells (PBMC) and synovial fluid mononuclear cells (SFMC) using flow cytometry.

\section{Methods}

SUBJECTS

Blood and synovial fluid was obtained from knee joints of outpatients $(n=28)$ who required arthrocentesis for therapeutic purposes. Sixteen subjects had rheumatoid arthritis and 12 had joint disease other than rheumatoid arthritis: psoriatic arthritis $(\mathrm{n}=2)$, seronegative spondarthropathy $(n=5)$, calcium pyrophosphate disease $(\mathbf{n}=2)$, osteoarthritis $(n=1)$, post-traumatic $(n=1)$ and post-streptococcal arthritis $(n=1)$. Informed consent was obtained.

\section{PROCEDURES}

Clinical assessment of the affected joint comprised pain (0-3), tenderness $(0 / 1)$, and 


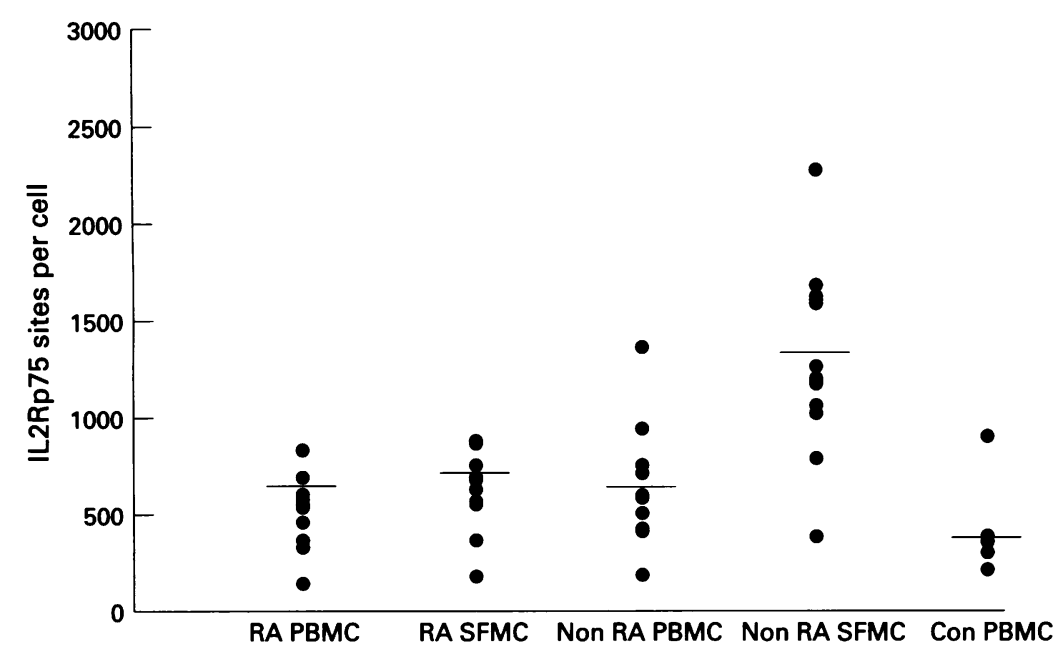

Figure 1 Quantitation of $I L-2 R$ p 75 sites.

synovitis (0/1). Plasma viscosity, $C$ reactive protein, and an articular index served as measures of systemic disease activity. Blood was also obtained from six control subjects (healthy volunteers).

SFMC and PBMC were isolated by differential centrifugation on a density gradient.

Indirect immunofluorescence staining was performed using an IgG1 monoclonal antibody directed against the p75 chain of the IL-2 receptor (TU27, kindly supplied by Dr J Hamuro, Ajinomoto Co, Japan). ${ }^{5}$ An irrelevant antibody of the same immunoglobulin subclass served as a negative control. Saturating concentrations of each were determined by titration experiments. Secondary antibody was rabbit antimouse immunoglobulin, $\mathrm{F}(\mathrm{ab})_{2}$ portion, FITC conjugated (Dako UK).

Double immunofluorescence staining of mononuclear cells was used to identify IL2Rp75 bearing cells as CD4 or CD8 positive and to allow an estimation of differential expression on these two cell subtypes. TU27 primary antibody, rabbit antimouse immunoglobulin, $\mathrm{F}(\mathrm{ab})_{2}$ portion, $\mathrm{R}-\mathrm{PE}$ conjugated secondary antibody (Dako UK), and conjugated CD4-FITC or CD8-FITC (Dako UK) antibodies were used. Samples were evaluated using a Facscan flow cytometer and Becton Dickinson LYSYS II software (Becton Dickinson UK). The lymphocyte population was established by position on a forward scatter:side scatter dot plot and confirmed by CD45:CD14 dual labelling. This population contained virtually all the detectable IL-2Rp75-positive cells. Quantitation of antigen was performed by calibration of the machine with standard beads expressing $2.8 \times$ $10^{3}$ to $33 \times 10^{3}$ FITC molecules per bead (low

Table 1 Binding sites for TU 27 antibody. Values are mean (SD)

\begin{tabular}{|c|c|c|c|c|c|}
\hline & $n$ & Age (years) & $\begin{array}{l}\text { Disease duration } \\
\text { (years) }\end{array}$ & PBMC IL-2Rp75 & $\begin{array}{l}\text { SFMC } \\
I L-2 R p 75\end{array}$ \\
\hline $\begin{array}{l}\text { RA } \\
\text { Non-RA } \\
\text { Controls }\end{array}$ & $\begin{array}{l}16 \\
12 \\
6\end{array}$ & $\begin{array}{l}59.2(16.9) \\
46.8(18.8) \\
52.0(7.5)\end{array}$ & $\begin{array}{l}9.1(8.4) \\
13.6(15.4) \\
\text { N/A }\end{array}$ & $\begin{array}{l}746(365) \\
783(368) \\
494(295)\end{array}$ & $\begin{array}{l}826(289) \\
1481(583)^{\star} \\
\text { N/A }\end{array}$ \\
\hline
\end{tabular}

RA, rheumatoid arthritis; PBMC, peripheral blood mononuclear cells; SFMC, synovial fluid mononuclear cells.

$\star P=0.0007$, SFMC RA $v$ non-RA.
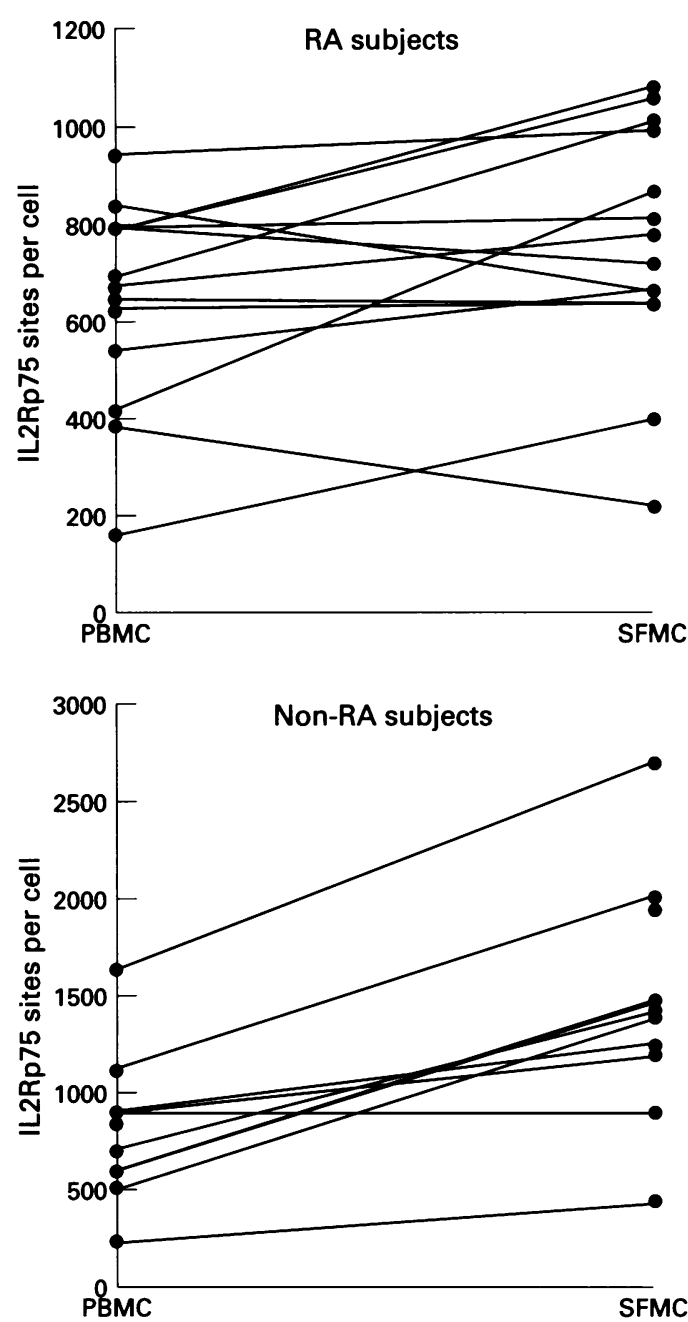

Figure $2 I L-2 R p 75$ sites on simultaneously obtained $P B M C$ and SFMC.

level quantitative fluorescein microbead standards, Flow Cytometry Standards Corporation). The FITC to protein $(\mathrm{F} / \mathrm{P})$ ratio was determined for each monoclonal antibody using "Simply Cellular Microbeads" (FCSC). Further details of the method have been described elsewhere. ${ }^{9}$

STATISTICAL METHODS

Values for binding sites had a normal distribution (Shapiro-Wilk test) and with over 12 subjects in each group, parametric statistics (Student's $t$ test) were used. Correlations were sought using a ranked Spearman correlation coefficient.

\section{Results}

Age and disease duration are shown in table 1. The index joint was tender in all subjects except one (osteoarthritis) and this was thought to be due to synovitis in all except four (two rheumatoid arthritis, one ankylosing spondylitis, one osteoarthritis). The mean pain score, graded 0-3, was 2.0 (SD 0.97) for rheumatoid arthritis and $2.2(0.7)$ for nonrheumatoid arthritis.

Binding sites detected by TU27 (anti IL-2Rp75) are shown in table 1 and fig 1 . There was no difference in IL-2Rp75 expression on PBMC from rheumatoid arthritis patients, non-rheumatoid arthritis patients, 
Table 2 Effect of disease duration on IL-2Rp75 expression. Values are mean (SD)

\begin{tabular}{llllllll}
\hline Disease duration & $n$ & $P V$ & CRP & AI & EMS (mins) & PBMC IL-2Rp75 & $S F M C$ IL-2Rp75 \\
\hline$\leq$ 3 years & 13 & $1.9(0.2)$ & $39.4(45.7)$ & $219(113)$ & $52.1(32.1)$ & $901(454)$ & $1237(595)$ \\
$>$ 3 years & 13 & $1.9(0.2)$ & $36.6(41.8)$ & $211(105)$ & $65.4(53.4)$ & $616(84)^{\star}$ & $953(473) \dagger$ \\
\hline
\end{tabular}

PV, plasma viscocity; CRP, C reactive protein; AI, articular index; EMS, early morning stiffness; PBMC, peripheral blood

mononuclear cells; SFMC, synovial fluid mononuclear cells.

* Mean PBMC ( $\leq 3$ years) $v$ ( $>3$ years $), P=0.048$.

† Mean SFMC ( $\leq 3$ years) $v$ ( $>3$ years $), \mathrm{P}=0.19$.

and controls. In rheumatoid arthritis subjects there was no difference in IL-2Rp75 expression on PBMC and SFMC. However, in the non-rheumatoid arthritis group there was an increase in IL-2Rp75 expression on SFMC compared with PBMC ( $\mathrm{P}=0.0032$ ) (fig 2). On SFMC there was a greater expression of IL-2Rp75 in non-rheumatoid arthritis compared with rheumatoid arthritis $(P=0.0007)$. Double labelling for $\mathrm{CD} 4$ or $\mathrm{CD} 8$ was performed on PBMC and SFMC from subjects with rheumatoid arthritis $(n=3)$ and non-rheumatoid arthritis $(n=3)$. PBMC were obtained from healthy controls $(n=3)$. The majority of cells in rheumatoid arthritis synovial fluid were CD4 positive $(65.8 \%$, compared with $27.9 \%$ CD8 positive), and an excess of CD4 cells was also seen in other diseases. Expression of IL-2Rp75 was associated with $\mathrm{CD} 8$ positive rather than $\mathrm{CD} 4$ positive cells.

The results were also analysed to examine the effect of disease duration (less than or more than three years) on expression of IL-2Rp75 (see table 2). The two groups were well matched in terms of plasma viscosity, C reactive protein level, articular index, and duration of early morning stiffness, but there was a greater expression of IL-2Rp75 in subjects with recent disease, significantly so in the case of PBMC. No correlation was seen between IL-2Rp75 levels and pain, plasma viscocity, $C$ reactive protein, articular index, or duration of early morning stiffness. Expression of IL2R p75 on PBMC correlated with expression on SFMC ( $r=0.44, P=0.02)$.

\section{Discussion}

We have re-examined IL-2R expression using antibody to the p75 chain and methodology which permits quantitation of receptor numbers. The methodology-like other methods of deriving antigen binding sites-make certain assumptions including most importantly, that binding of antibody to surface of bead and surface of cell will be similar. Errors introduced as a result of this will be similar for all cells, and conclusions about relative (rather than absolute) antigen binding capacity are therefore likely to remain valid. Quantitation of IL-2Rp75 sites gave values consistent with those obtained using traditional methods (180-410 sites per resting CD8 cell). ${ }^{5}$

We found no difference in expression of IL-2Rp75 on PBMC between controls and subjects with inflammatory arthritis. While levels for all subjects were increased within synovial fluid, this increase was only significant for non-rheumatoid arthritis subjects, and expression of IL-2 R p75 was significantly higher on non-rheumatoid arthritis SFMC than on rheumatoid arthritis SFMC. The failure to detect higher levels of IL2Rp75 in the rheumatoid arthritis joint was not due to a lack of cells capable of upregulation. IL-2Rp75 was expressed on CD8 $\mathrm{T}$ cells which comprised about a quarter of all lymphocytes in the joint. CD4 lymphocytes were also abundant in rheumatoid joints and, while resting CD4 cells express only low levels of IL-2Rp75, those from normal individuals are capable of upregulation when stimulated by, for example, PHA. ${ }^{5}$ This does not seem to be occurring in the rheumatoid joint.

Subjects with rheumatoid arthritis had a longer disease duration than the nonrheumatoid arthritis group. It is probable that the factors responsible for initiating rheumatoid arthritis are different from those which maintain it. When the effect of disease duration was examined, higher levels of IL-2Rp75 were found in early disease, but even "early" rheumatoid arthritis patients expressed less IL-2Rp75 within the joint than nonrheumatoid arthritis patients of greater disease duration.

Impaired upregulation of IL-2Rp75 on rheumatoid arthritis synovial $\mathrm{T}$ cells implies a lack of appropriate stimulation in this environment (disease related or otherwise) or inhibition through some suppressive mechanism. Both clinical and laboratory assessments of disease activity failed to show any difference between rheumatoid arthritis and nonrheumatoid arthritis patient groups in this study. Significant levels of class II MHC expression have been well documented on rheumatoid arthritis SFMC, ${ }^{10}$ and recent studies have also detected the costimulatory molecule B7.2 (CD86) in rheumatoid synovium. ${ }^{11}$ This suggests that appropriate molecules for $T$ cell activation are expressed in the rheumatoid joint. It is possible, however, that the activated cells within the rheumatoid synovium are there simply because they are the only cells possessing adhesion molecules to allow them to escape from the circulation. ${ }^{12}$ The increase in IL-2 receptor expression within the joint in the non-rheumatoid group would imply further local activation of $T$ cells (for example, by bacterial products) that is not occurring in rheumatoid arthritis. Finally, an inhibitor or suppressor mechanism may exist in the rheumatoid joint. Biologically active IL-2 is low in rheumatoid arthritis though IL-2 mRNA is present in abundance. ${ }^{13}$ Surface thiol groups on mononuclear cell membranes are involved in cellular responses to exogenous IL-2 and expression of both IL-2Rp55 and IL-2Rp75. ${ }^{14}$ Oxidation of thiol groups on 
rheumatoid arthritis SFMC could interfere with $T$ cell activation resulting in impaired upregulation of IL2Rp75. This implies that unique conditions exist in the rheumatoid joint, capable of exerting significant oxidative stress. $^{15}$

The impaired function of synovial $\mathrm{T}$ cells, as represented by a relative reduction in IL-2Rp75 expression, could result in failure to regulate proinflammatory functions of non-T cells in the joint, leading to uncontrolled, cytokine driven, chronic inflammation with the clinical and pathological sequelae seen in established rheumatoid arthritis.

1 Panayi GS, Lanchbury JS, Kinsley GH. The importance of the $T$ cell in initiating and maintaining the chronic synovithe T cell in initiating and maintaining the chronic synovi-
tis of rheumatoid arthritis. Arthritis Rheum 1992;35:729tis of

2 Firestein GS, Zvaifler NJ. How important are T cells in chronic rheumatoid synovitis? Arthritis Rheum 1990; 33:768-73.

3 Pitzalis C, Kingsley G, Lanchbury JSS, Murphy J, Panayi GS. Expression of HLA-DR, DQ and DP antigens and interleukin 2 receptor on synovial fluid $T$ lymphocyte subsets in rheumatoid arthritis: evidence for "frustrated activation". $\mathcal{f}$ Rheumatol 1987;14:662-6.

4 Lotz M, Tsoukas CD, Robinson CA, Dinarello CA, Carson DA, Vaughan JH. Basis for defective responses of rheumatoid arthritis synovial fluid lymphocytes to anti-CD3 (T3) toid arthritis synovial fluid lymphocytes to
antibodies. $\mathcal{F}$ Clin Invest 1986;78:713-21.

5 Ohashi Y, Takeshita T, Nagata K, Mori S, Sugamura K. Oifferential expression of the II-2 receptor subunits, p55
Dita and p75 on various populations of primary peripheral blood mononuclear cells. F Immunol 1989;143:3548-55.

6 Tsudo M, Goldman CK, Bongiovanni KF, Chan WC, Winton EF, Yagita M, et al. The p75 peptide is the receptor for interleukin 2 expressed on large granular lymphocytes and is responsible for the interleukin 2 activation of these cells. Proc Natl Acad Sci USA 1987;84:5394-8.

7 Kasahara T, Hooks JJ, Doughtery SF, Oppenheim JJ. Interleukin 2 mediated immune interferon (IFN gamma) production by human T cells and T cell subsets. F Immunol 1983;130:1784-9.

8 Hasler F, Dayer J-M. Diminished II2 induced gamma interferon production by unstimulated peripheral blood interferon production by unstimulated peripheral blood 27:15-20.

9 Creamer P, Edmonds J, Sullivan J, Matthews S. Measurement of HLA class 1 expression in ankylosing spondylitis. Ann Rheum Dis 1992;51:1138-42.

10 Burmester GR, Yu DTV, Irani AM, Kunkel HC, Winchester RJ. Ia and T cells in synovial fluid and tissues of patients with RA. Arthritis Rheum 1981;24:1370-6.

11 Balsa A, Dixey J, Sansom DM, Maddison PJ, Hall ND. Differential expression of the costimulatory molecules B7.1 (CD80) and B7.2 (CD86) in rheumatoid synovial tissue. Br $\mathcal{F}$ Rheumatol 1996;35:33-7.

12 Ziff $M$. Role of the endothelium in chronic inflammatory arthritis. Arthritis Rheum 1991;34:1345-52.

13 Howell WM, Warren CJ, Cook NJ, Cawley MID, Smith JL. Detection of IL 2 at mRNA and protein levels in synovial infiltrates from inflammatory arthropathies using biotinylated oligonucleotide probes in situ. Clin Exp Immunol 1991;86:393-8.

14 Smith S, Brown-Galatola CH, Hall ND. Modulation of human $T$ cell functions by surface sulphydryl groups: differential effects on II 2 production and responsiveness. Clin Exp Immunol 1992;88:169-73.

15 Halliwell B. Oxygen radicals, nitric oxide and human inflammatory joint disease. Ann Rheum Dis 1995;54:50510 